The Journal of Animal \& Plant Sciences, 30(5): 2020, Page: 1145-1153

ISSN (print): 1018-7081; ISSN (online): 2309-8694

\title{
POMEGRANATE JUICE AMELIORATES ATENOLOL TOXIC EFFECTS ON EMBRYO DEVELOPMENT
}

\author{
S. Zafar ${ }^{1}$, Asmatullah $^{1}$ and C. Ara ${ }^{1, *}$ \\ ${ }^{1}$ Department of Zoology, New Campus, University of the Punjab, Lahore, Pakistan \\ "Corresponding author’s email: dr.chamanara@yahoo.com; chaman.zool@pu.edu.pk
}

\begin{abstract}
Hypertension is an increasing issue experienced by females during pregnancy. Gestational hypertension is treated by many antihypertensive drugs including methyldopa, beta blockers, and calcium channel blockers. Atenolol is usually the drug of choice by obstetricians among beta blockers. Objective of this study was to find the fetotoxic effects of Atenolol in albino mice and protective potential of pomegranate juice against potential atenolol toxicity. Embryotoxic effect of atenolol was determined in pregnant mice, force fed orally through gavage three different atenolol concentrations $(3.30 \mu \mathrm{g} / \mathrm{g}, 2.50 \mu \mathrm{g} / \mathrm{g}, 1.65 \mu \mathrm{g} / \mathrm{g}$ body weight of treated mice, respectively) from 6th -12th day of gestation. Other three groups were made for antidote study and 50\% diluted pomegranate juice was provided to each group along with above mentioned atenolol concentrations as an antidote. During osteogenesis, fetuses in atenolol exposure groups have varying degree of incomplete ossification in dose dependent way. Histological analysis had also shown teratogenic potential of atenolol in developing mice embryos. The present study revealed that administration of atenolol during organo-genetic period upset prenatal development in mice fetuses. To minimize the toxic effects of drug, pomegranate juice was provided to experimental mice. Positive outcomes represented that pomegranate juice has protective potential against Atenolol induced fetotoxicity.
\end{abstract}

Key words: Atenolol, Fetotoxity, Hypertension, Ameliorative, Pomegranate.

https://doi.org/10.36899/JAPS.2020.5.0131

Published online June 25, 2020

\section{INTRODUCTION}

Hypertension during pregnancy is one of the major problems faced by obstetricians (Andrade et al., 2004). A recent study in United Kingdom reported that about one-third of the total maternal morbidity was caused by hypertensive disorders. Another study by National institute for health and care excellence stated that 1 out of 20 women with severe hypertension during pregnancy were admitted to hospital (Alexander \& Wilson, 2013; McCormack et al., 2012). Many disorders caused by hypertension in pregnancy bear risks for the woman and the baby. It is a significant cause of morbidity and mortality during pregnancy. Recent indication by few clinical trials had revealed that medicinal management is beneficial for the reduction of hypertension and other outcomes related to the hypertension (James et al., 2014).

There are a lot of antihypertensive drugs available that belong to different classes. In Churchill's pocketbook of hypertension, beta-blockers are said to be appropriate as initial choice for young patients in early phase of hypertension in non-obstetric patients as well as pregnancy-associated hypertension (Isla et al., 2005; Hind and Sara, 2014). Among beta-blockers, atenolol is a commonly used antihypertensive drug during pregnancy (Butters et al., 1990). So in pregnancy the risk of preeclampsia and eclampsia is greatly reduced in cases where atenolol is used as antihypertensive treatments (Lydakis et al., 1999).

Some reports of atenolol related fetopathy in newborns were published in last few decades. These reports were featuring few individual case reports where mothers were treated for hypertension (Freyer, 2009). However, there is a lack of systemized data. Keeping in view this deficiency; it seems unavoidable to conduct experiments on animal models. In NICE clinical guidelines it is advised that the expected benefits should be measured against potential threats of drug use in pregnancy. Informed approval on the use of atenolol in these situations should be acquired and documented (Moussa et al., 2014).

Antihypertensive medication is indicated if the systolic blood pressure rises above $150-160 \mathrm{mmHg}$ or diastolic blood pressure rises above $100-110 \mathrm{mmHg}$ or there is any end organ damage (Chobanian et al., 2003). Atenolol remained the drug of choice for the last few decades. Its wide range uses, success and comparatively less side effects have added to its reputation. Furthermore, the absence of severe metabolic alterations enhanced its value as antihypertensive (Mancia et al., 2009). The reports of atenolol related fetotoxicity in newborns revealed its major drawback (Reynolds et al., 1984). Present study was conducted to evaluate fetotoxity of Atenolol and to minimize its effects by using pomegranate juice as a natural remedy for improving 
maternal and fetal health. As our research is on mammalian model (mice) so results can be extrapolated to humans.

\section{MATERIALS AND METHODS}

Swiss Webster male and female albino mice of 6-week old were taken from Veterinary Research Institute, Lahore, Pakistan, having weight about $28 \pm 2 \mathrm{~g}$. They were put into sanitized steel cages (1 male:2 female) and were allowed to mate freely for raising colony. Mice were placed in animal house with good ventilation and maintained temperature $\left(27 \pm 2^{\circ} \mathrm{C}\right)$. Cages were well supplied with water and mice Feed \# 13, manufactured by National Feeds Ltd., Lahore, Pakistan. Mated females were recognized by the presence of semen as white colored plug. The date was noted as day of conception. The next day is counted as first day of gestational period. The pregnant mice were then placed in separate cages to avoid any interference.

Experimental design and dose administration: Mice were randomly divided into 8 groups designated as control (C), vehicle control (VC) and 3 atenolol treated (LD), (MD), (HD) and 3 atenolol + antidote treated groups $(\mathrm{LD}+\mathrm{AD}),(\mathrm{MD}+\mathrm{AD}),(\mathrm{HD}+\mathrm{AD})$. Each group contains 10 pregnant females. Different concentrations of atenolol were prepared in such a way that $0.1 \mathrm{ml}$. of solution contained the desired amount of drug. The high dose contains Atenolol as $3.30 \mu \mathrm{g} / \mathrm{g}$ body weight (B.wt.) of treated mice, medium dose contains $2.50 \mu \mathrm{g} / \mathrm{g}$ B.wt. and low dose contain $1.65 \mu \mathrm{g} / \mathrm{g}$ B.wt. In Atenolol treated groups females were forced fed and desired concentrations were administered orally via gavage from 6 th -12 th day of gestation regularly once in a day.

Fresh pomegranates (Punica granatum) were bought from local market and washed carefully to remove surface adherents. The peel of pomegranates was removed and the seeds were crushed by using blender. The residue was passed through filter paper no.40 (Whatmans) and juice was obtained. The juice collected was prepared by adding distilled water in $50 \%$ proportion (Awari et al., 2009).

Three groups were made for antidote study and $50 \%$ diluted pomegranate juice was provided to each high (3.30 $\mu \mathrm{g} / \mathrm{g}$ B.wt.), medium (2.50 $\mu \mathrm{g} / \mathrm{g}$ B.wt.) and low dose (1.65 $\mu \mathrm{g} / \mathrm{g}$ B.wt) group (HD+AD), (MD+AD), $(\mathrm{LD}+\mathrm{AD})$ along with the drug dose from $6^{\text {th }}-12^{\text {th }}$ gestational day as a sole treatment. Each mouse approximately consumes $5 \mathrm{ml}$ pomegranate juice in a day.

Fetal Skeletal analysis: Method of Kawamura et al. (1990) was followed to prepare fetal skeleton. The selected fetuses from exposure groups and antidote groups were given an abdominal incision and viscera were removed. Then fetuses were placed in $2 \% \mathrm{KOH}$ for clearing flesh from bones. 2-3 drops of $1 \% \mathrm{KOH}$ were added to Alizarin Red $\mathrm{S}$ and the solution is used to stain fetuses. $20 \%$ glycerinated $1 \% \mathrm{KOH}$ was used to enhance visibility and then specimens were preserved in 50\% ethanolic glycerol. Macro-photography was done by using Panasonic Lumix TZ15 camera.

Fetal Histological analysis: All females were anaesthetized with 5\% isoflurane inhalation and dissected at $18^{\text {th }}$ gestational day and fetuses were fixed in Bouin's fixative for further studies (Baker, 1958). Some fetuses were selected from all dose groups for histological studies including control and VC groups. Fetuses after fixation in Bouin's fixative were washed with 70\% alcohol several times to completely remove the color of picric acid. Then dehydrated with higher grades of ethanol i.e. $70 \%, 90 \%$ and $100 \%$ gradually. Fetuses were left over night in xylene for clearance and then immersed in paraffin wax for infiltration.

Fetuses embedded in molten paraffin wax in special molds for the process of section cutting. After solidification, molds were removed and wax blocks were trimmed. These blocks with embedded fetuses were then subjected to microtome for cutting $4-5 \mu$ thick transverse sections. To reduce the wrinkles, these sections were spread in water bath with warm water at $37-40^{\circ} \mathrm{C}$. Egg albumin was coated on slides before mounting the sections. These slides were stained with hematoxylin, and eosin was used as a counter stain. Canada balsam was applied on stained sections for microbial protection and for clearance. All slides were then studied using stereoscopic compound microscope following atlas of mouse development (Matthew H. Kaufman \& Kaufman, 1992) to observe congenital anomalies. Microphotographs were taken by using digital camera.

\section{RESULTS AND DISCUSSION}

Skeletal Studies: The structures of all specimens from control and vehicle control group were well ossified and stained (Fig.1).

Fetuses in atenolol exposure groups have less ossified skeleton in carpals, metacarpals, phalanges, tarsals and metatarsals. In high dose group ossification of forelimb and hind limb was also affected (Figs. 2\& 3). Simple arrows showed less degree of ossification while bold arrows showed no ossification in three different dose groups as well as in antidote groups. Similar decrease in skeletal ossification and length of umbilical cord was also observed previously along with structural deviations indicate intrauterine growth retardation (Tabacova et al., 2003). Fitzgerald reported that renal pelvic enlargement was also observed in some cases (Fitzgerald et al., 1978). The damaging oxidative effect of low density lipids is minimized by Polyphenolic flavonoids in pomegranate juice which inhibits the development of atherosclerosis (Aviram \& Rosenblat, 2012). 
Lablels: F: Frontal, N: Nasal, Pm: Pre Maxila, Md: Mandible, H: Humerus, R: Radius, U: Ulna, Ri: Ribs, Ti:
Tibia, Fi: Fibula, Fe: Femur, A: Atlas, Eo: Exocipital, So: Supraocipiltal, Ip: Interperitonial, P: Parietal
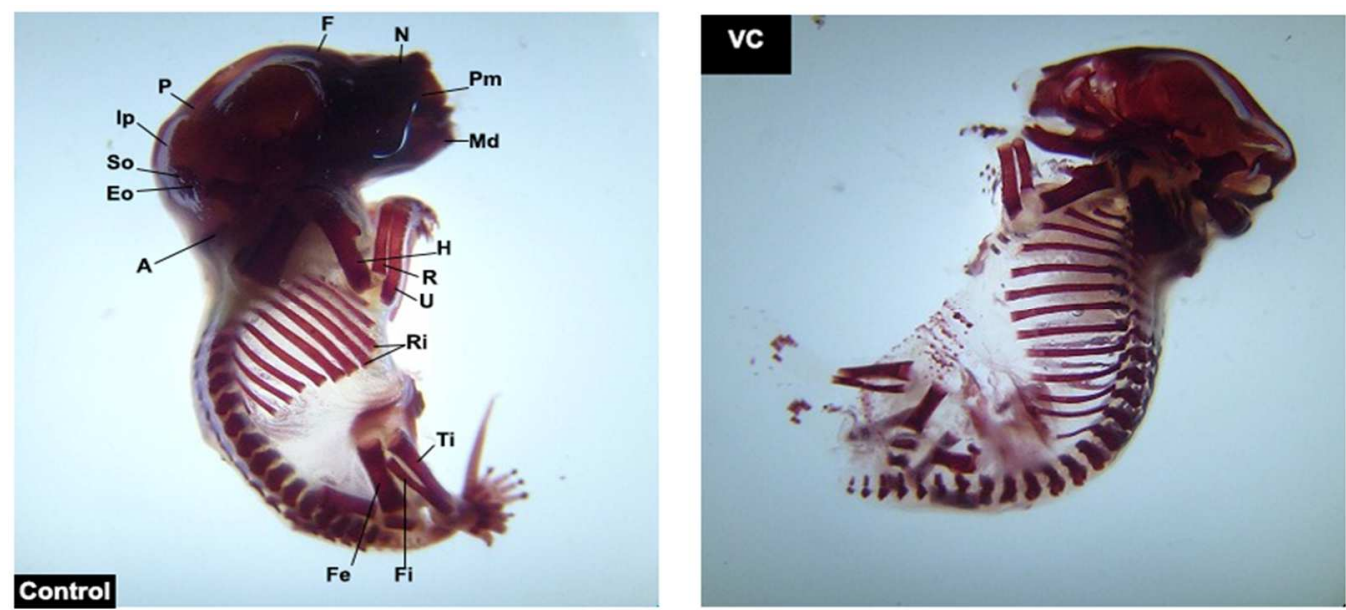

Figure 1: Fetuses obtained from Control and Vehicle Control Group showing well ossified skeleton.
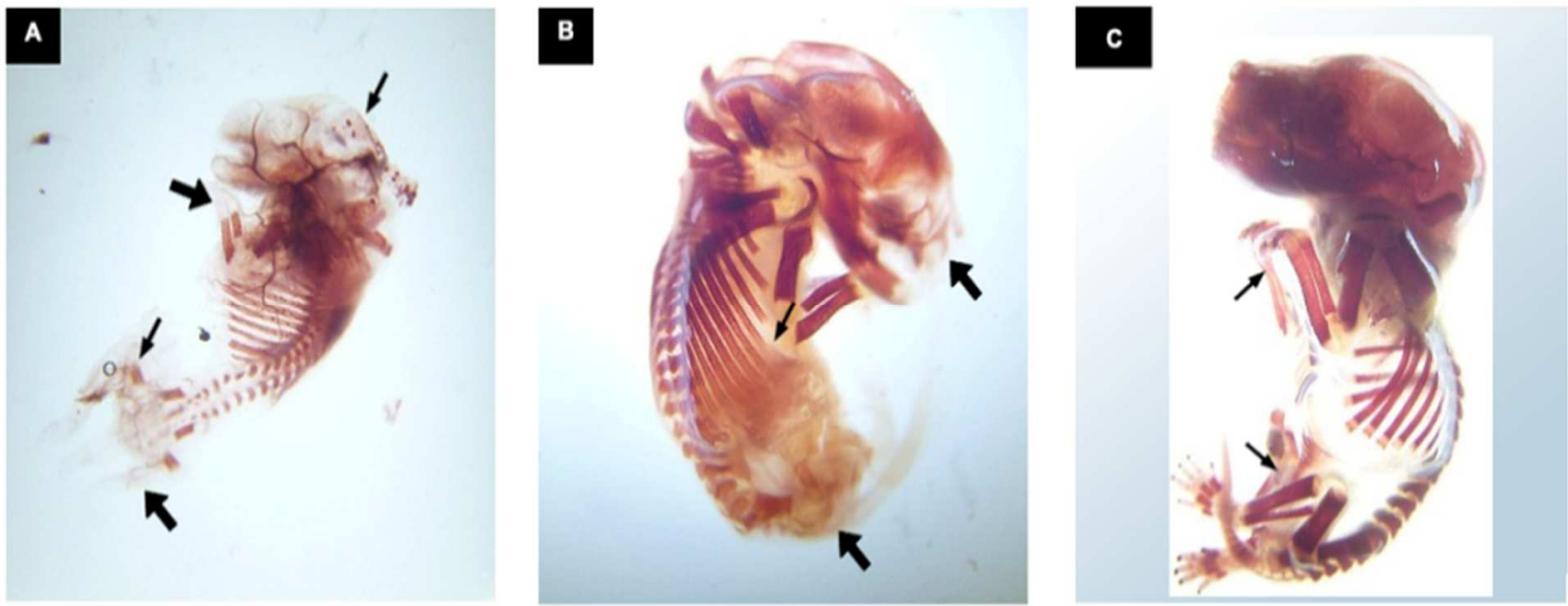

Figure 2: Fetuses obtained from mothers administered with atenolol showing defective and less ossified skeleton [A: High dose, B: Medium dose, C: Low dose]
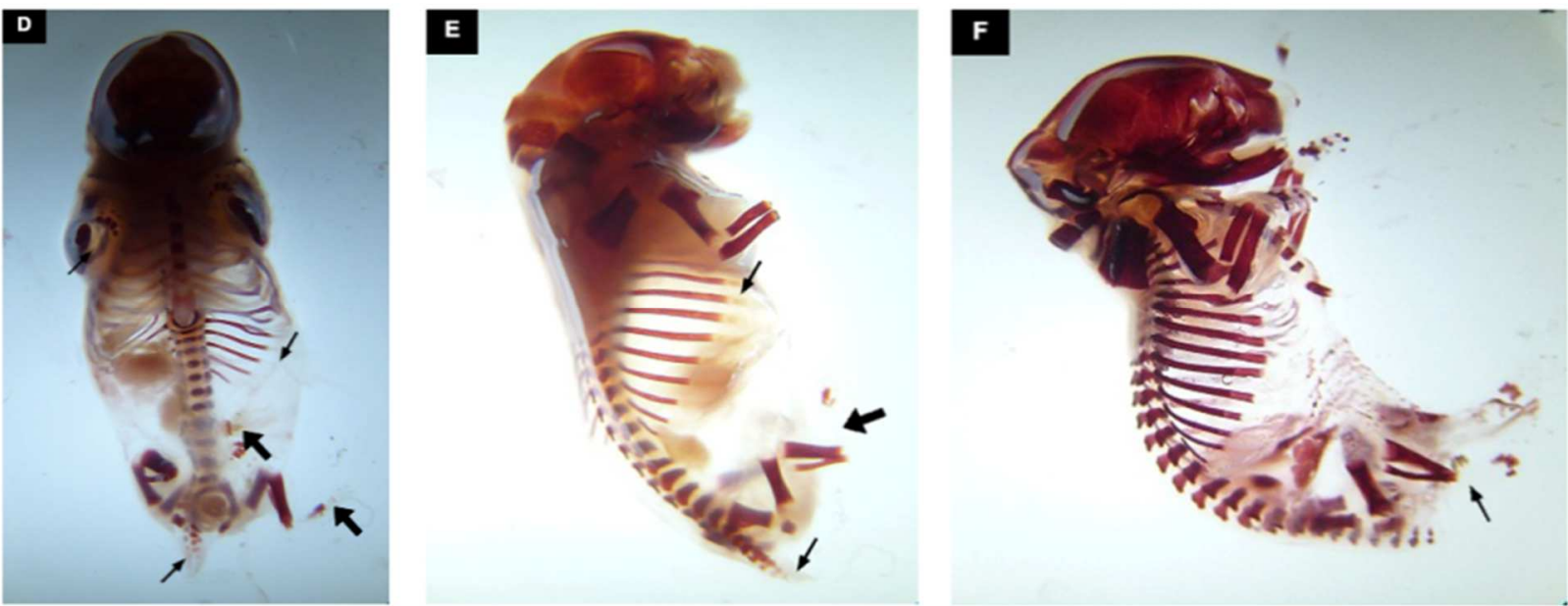

Figure 3: Fetuses obtained from mothers administered with atenolol + pomegranate juice clearly indicating protective effect of pomegranate juice [D: High dose+ AD; E: Medium dose + AD ; F: Low dose+ AD]. 
Histological studies: Histological studies of major fetal viscera including spinal cord, lung, liver, heart and brain was done to understand histopathological changes caused by atenolol. For histological studies some fetuses were randomly selected from each group.

Control: All fetuses belong to control group depicted well developed structures with normal features as shown in fig. 4

Vehicle Control (VC): Fetuses in the vehicle control group were normal and have quite developed cardiac and other viscera (fig. 5)

Low Dose Group (1.65 $\mu \mathrm{g} / \mathrm{g}$ B.wt): This exposure group had fetuses with normal structures a few histological defects like misshapen or distorted structures were observed in few cases (fig. 6)
Medium Dose Group (2.50 $\mu \mathrm{g} / \mathrm{g}$ B.wt.): Middle dose group had fetuses with internal defects like misshapen and with mal positioned body structures. In figure shown below undifferentiated neuroglial cells in the lateral part of cerebellum and herniation in $4^{\text {th }}$ ventricle was obvious (Fig. 7). In heart region, hypoplasia of atrium and thick walled ventricles were observed.

High Dose Group (3.30 $\mu$ g/ g B.wt.): High dose group had fetuses with internal defects like reduced sized organs, degenerated muscles was observed. Enlargement of fourth ventricle, degeneration of submandibular gland on right side was also clear in fig. 8.

Developmental toxicity of atenolol is assumed previously and different researchers have established more or less similar findings in this regard (Bayliss et al., 2002; Lip et al., 1997; Lydakis et al., 1999; Tabacova et al., 2003).
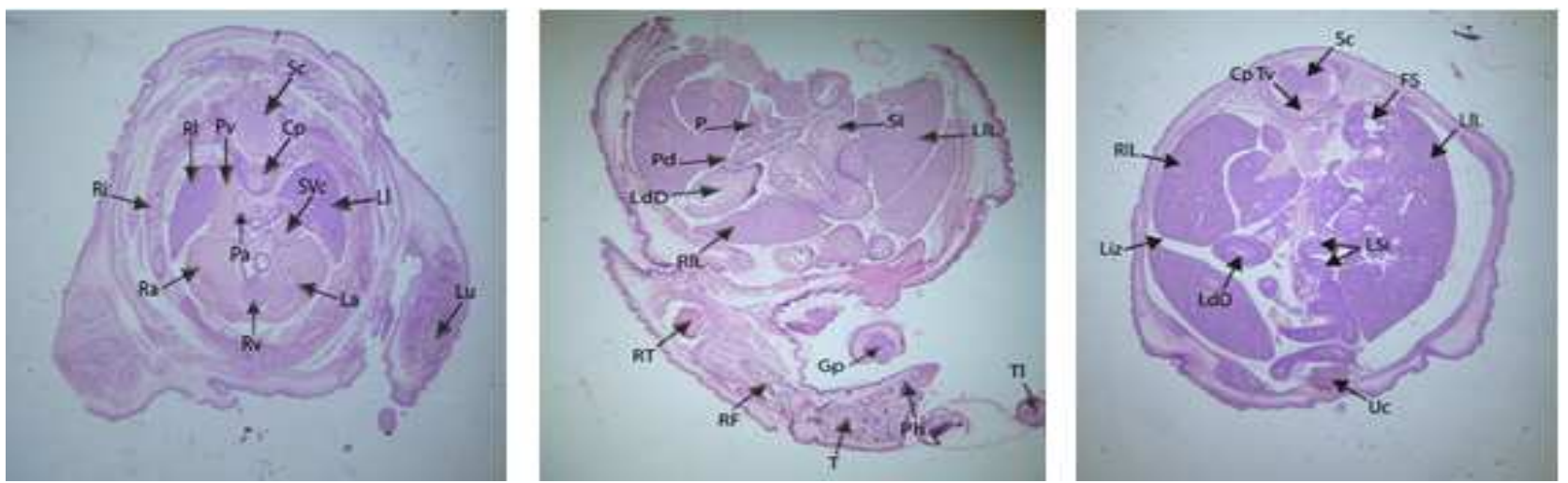

Figure 4: Histological sections of control group fetuses through cardiac and hepatic regions

Labels: Sc: Spinal Cord, Cp: Cartilage Primordium of Basisphenoid, SVc: Superior Vena Cava, Ll: Left Lung, La: Left Atrium, Lu: Left Ulna, Rv: Right Ventricle, Pa: Pulmonary Artery, Pv: Pulmonary Vein, Rl: Right Lung, Ri: Ribs, Ra: Right Atrium, Fs: Fundus Region of Stomach, LlL: Left Lobe of Liver, Vt: Vagal Trunk, IVc: Inferior Vena Cava, RcD: Right Crus of Diaphragm, CpTv: Catilage Primordium of Upper Thoracic Vertebral
Body, RIL: Right Lobe of Liver, Liz: Lobar Inter Zone, LdD: Lumen of Decending part of Duodenum, LSi: Lumen of Small Intestine, Uc: Umbilical Cord, D: Diaphragm, Pv: Portal Vein, P: Pancreas, Pd: Pancreatic Duct, Si: Small Intestine, RT: Right Tibia, Pp: Prepuce, Gp: Glans Penis, Rf: Right Fibula, T: Tarsus, $\mathrm{Ph}$ : Phalanges, Tl: Tail, Tc: Tentorium Cerebelli,
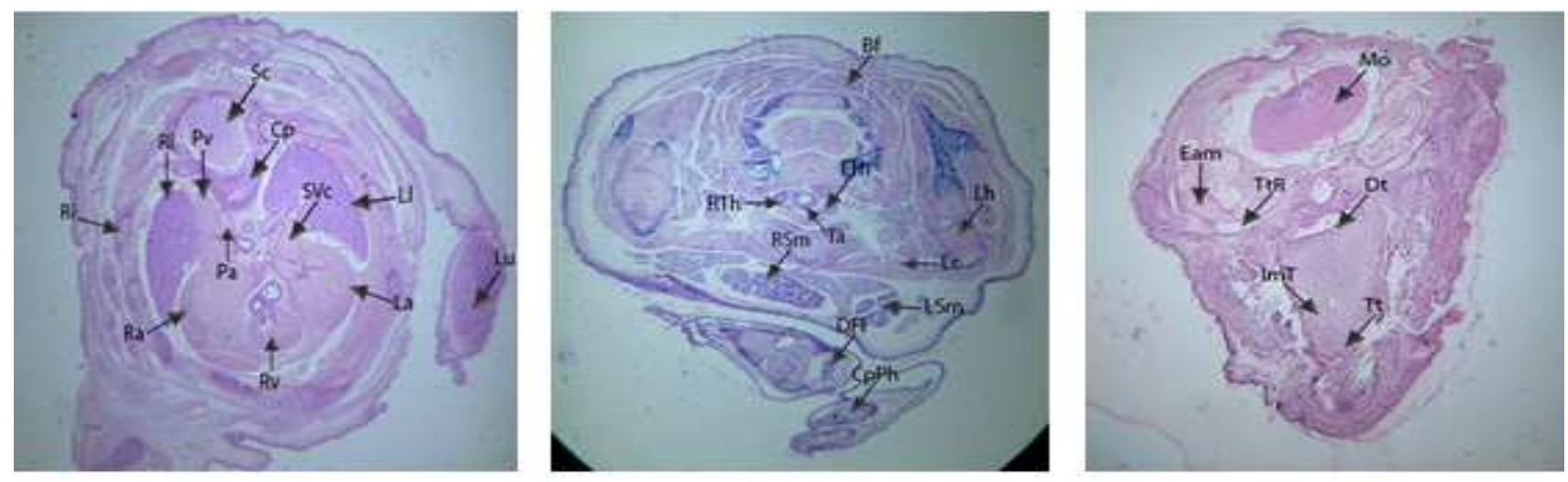

Figure 5: Histological sections of vehicle control group fetuses through cardiac and sublingual regions 
Labels: Sc: Spinal Cord, Cp: Cartilage Primordium of Basisphenoid, SVc: Superior Vena Cava, Ll: Left Lung, La: Left Atrium, Lu: Left Ulna, Rv: Right Ventricle, Pa: Pulmonary Artery, Pv: Pulmonary Vein, Rl: Right Lung, Ri: Ribs, Ra: Right Atrium, Bf: Brown Fat, Ml: Mantle Layer, Ft: Foramen Transversarium of Cervical Vertebra, Ls: Left Scapula, Cc: Cricoid Cartilage, LTh: Left Lobe of Thyroid Gland, Lh: Left Humerus, Tr: Trachea, ItG: Intertubercular Groove, RTh:
Right Lobe of Thyroid Gland, RSm: Right Submandibular gland, Rc: Right Clavicle, Lc: Left Clavicle, LSm: Left Submandibular gland, DFI: Digit of Right Forelimb, $\mathrm{CpPh}$ : Cartilage Primordium of Phalangeal Bones, $\mathrm{Cm}$ : Constrictor Muscle, $\mathrm{Cp}$ : Cartilage Primordium of Basisphenoid, Dt: Dorsum of Tongue, Eam: External Auditory Meatus, ImT: Intrinsic Muscle of Tongue, Tt: Tip of Tongue, TtR: Tubo - Tympanic recess.
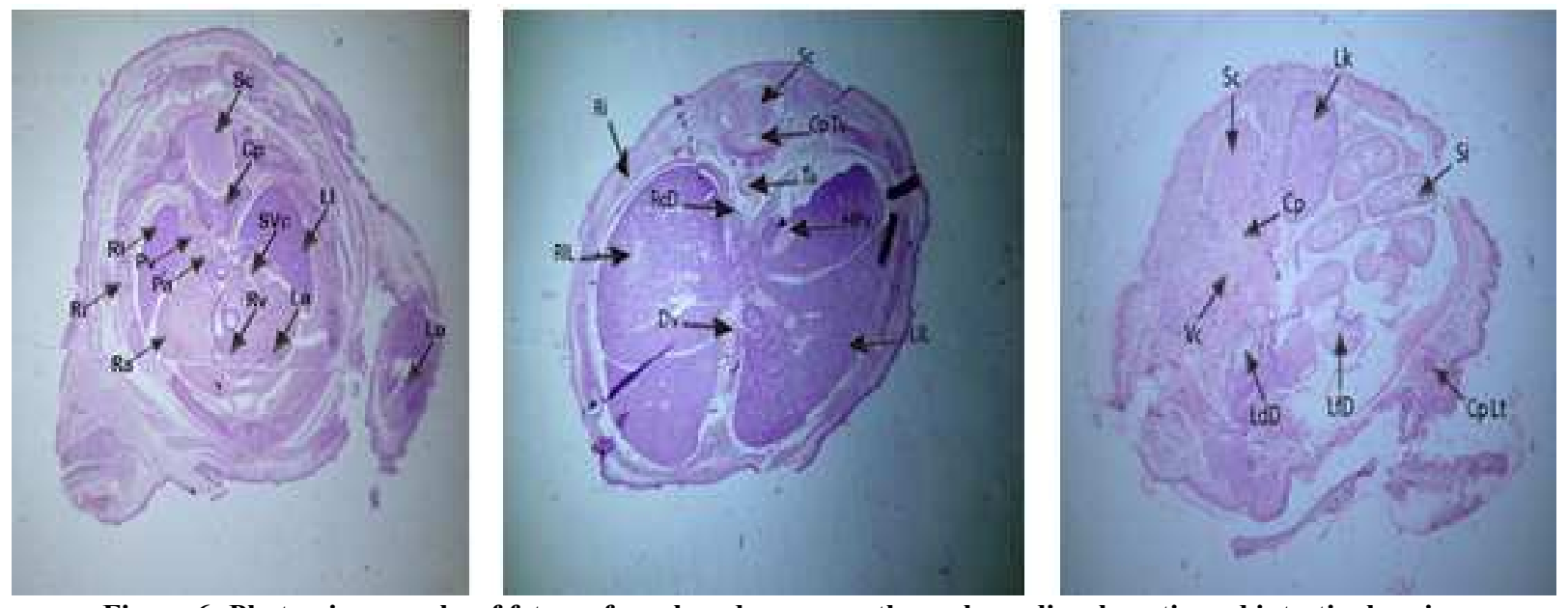

Figure 6: Photomicrographs of fetuses from low dose group through cardiac, hepatic and intestinal region

Labels: Cp: Cartilage Primordium of Basisphenoid, La: Left Atrium, Ll: Left Lung, Lu: Left Ulna, Pa: Pulmonary Artery, Pv: Pulmonary Vein, Ra: Right Atrium, Ri: Ribs, Rl: Right Lung, Rv: Right Ventricle, Sc: Spinal Cord, SVc: Superior Vena Cava, Cp: Cartilage Primordium of Basisphenoid, CpLt: Cartilage Primordium of Left Femur, LdD: Lumen of Decending part of Duodenum,
LfD: Lumen of First part of Duodenum, Lk: Left Kidney, Sc: Spinal Cord, Si: Small Intestine, Vc: Vena Cava, CpTv: Catilage Primordium of Upper Thoracic Vertebral Body, Dv: Ductus Venosus, HPv: Hepatic Portal Vein, LlL: Left Lobe of Liver, Ri: Ribs, RlL: Right Lobe of Liver, LcD: Left Crus of Diaphragm, Sc: Spinal Cord, Ta: Trachea.
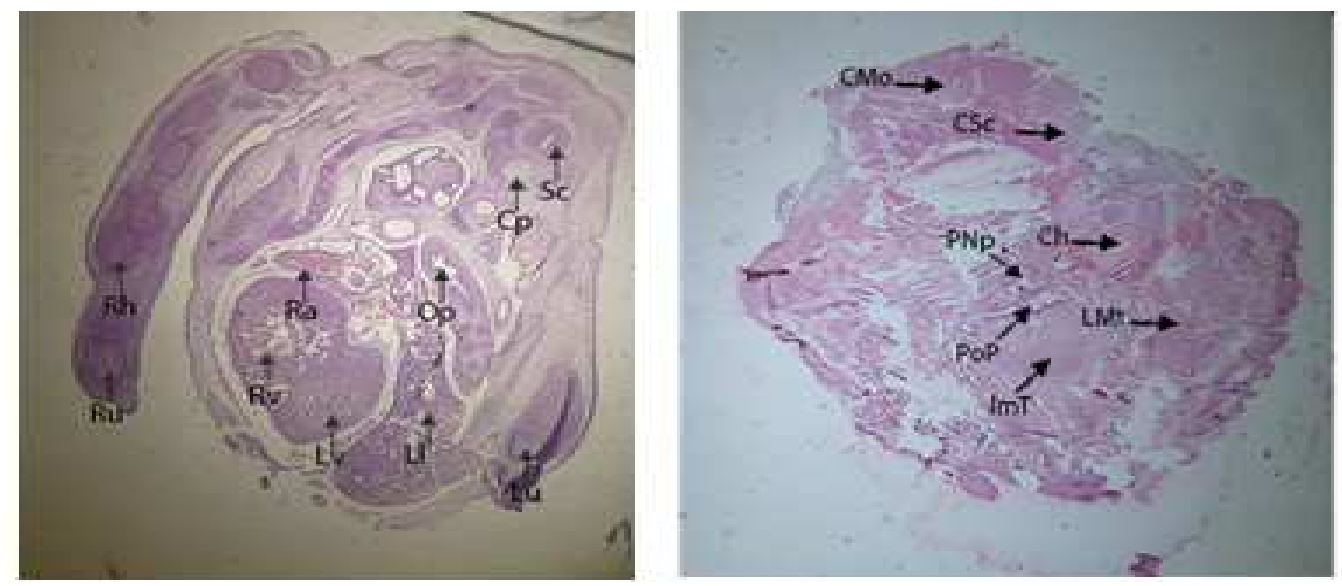

Figure 7 : Photomicrographs of fetuses from medium dose group through cardiac and sublingual regions.

Labels: Cp: Cartilage Primordium of Basisphenoid, Ll: Left Lung, Lv: Left Ventricle, Op: Esophagus, Ra: Right Atrium, Rh: Right Humerus, Ru: Right Ulna, Rv: Right Ventricle, Sc: Spinal Cord, CMo:
Caudal part of Medulla Oblongata, CSc: Clivus, ImT: Intrinsic Muscle of Tongue, PNp: Posterior Nasopharynx, POp: Posterior Part of Pharynx, LMt: Left Molar Tooth, Ch: Cochlea 

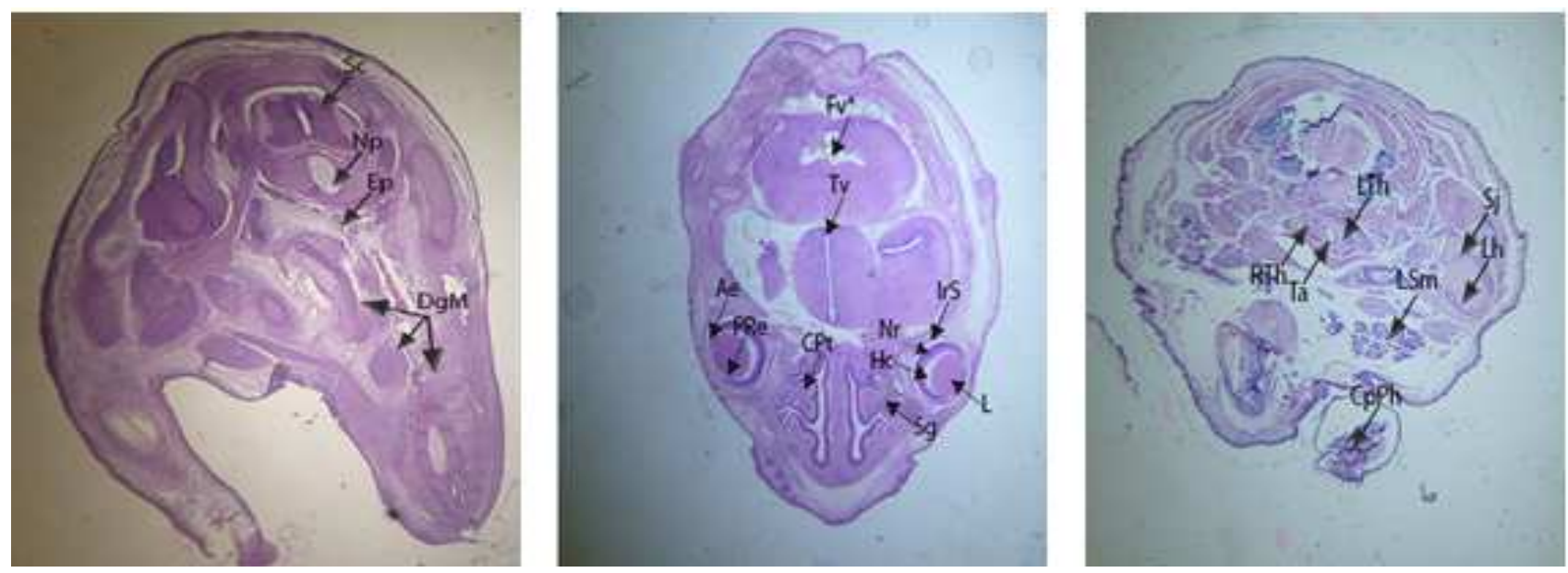

Figure 8: Photomicrographs of fetuses from high dose group brain region.

Labels: DgM: Degenerated Muscles, Np: Nucleus Pulposus, Sc: Spinal Cord, Fv*: Fourth Ventricle, Tv: Third Ventricle, CpPh: Cartilage Primordium of Phalangeal Bones, Lh: Left Humerus, LSm: Left Submandibular gland, LTh: Left Lobe of Thyroid Gland, RTh: Right Lobe of Thyroid Gland, Sj: Shoulder Joint, Ta: Trachea, Sc: Spinal Cord, Fv: Fourth Ventricle, Hc: Hyaloid Cavity, IrS: Intra Retinal Space, L: Lens, Nr: Neural Layer of Retina, PRe: Pupil of Right Eye, Sg: Serous Gland, Tc: Tentorium Cerebelli, Tv: Third Ventricle.

Low Dose (1.65 $\mu \mathrm{g} / \mathrm{g}$ B.wt) + Antidote Group: This dose group has similar results to control and vehicle control groups (fig. 9), with normal and well developed fetuses.

Medium Dose (2.50 $\mu \mathrm{g} / \mathrm{g}$ B.wt.) + Antidote Group: This group also shows abnormalities like mishappened structures (fig. 10). Poorly formed ventricular chambers and degeneration of cardiac muscles especially in atrium was observed.

These results are comparable with the other studies. The fruit juice of punica granatum is reported for cardiotonic activity. Studies demonstrated positive inotropic activity of pomegranate extract on isolated frog's heart (Ravindra et al., 2012). Pomegranate juice used as an antidote in present study significantly minimized the detrimental effects of atenolol during pregnancy. Recent clinical trials have demonstrated many advantages of pomegranate juice consumption. Pomegranate contains polyphenols and anthocyanins which are beneficial to cardiac health as these compounds improve vascular function and have anti-inflammatory effects (Aviram et al., 2004).
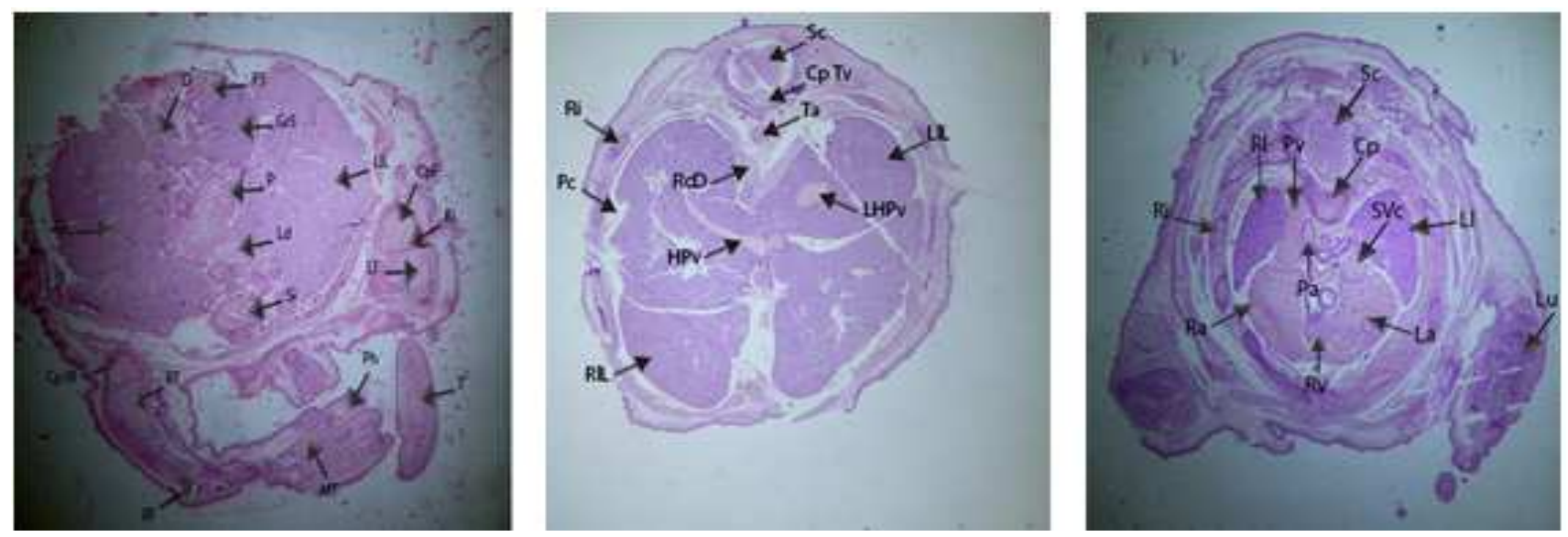

Figure 9: Photomicrographs of fetuses from low dose + antidote group through hepatic and cardiac regions

Labels: Cp: Cartilage Primordium of Basisphenoid, La: Left Atrium, Ll: Left Lung, Lu: Left
Ulna, Sc: Spinal Cord, Pa: Pulmonary Artery, Pv: Pulmonary Vein, Ra: Right Atrium, Ri: Ribs, Rl: Right 
Lung, Rv: Right Ventricle, SVc: Superior Vena Cava, Ae: Anterior Chamber of Eye, CPt: Cartilage Primordium of Turbinate Bone, DVs: Transverse Dural Venous Sinus, RcD: Right Crus of Diaphragm, Ri: Ribs, RlL: Right Lobe of Liver, Sc: Spinal Cord, Ta: Trachea, CpF: Cartilage Primordium of Femur, Cp RF: Cartilage Primordium of Right Femur, D: Diencephalon, FS: Fundus Region of Stomach, GrS: Glandular Region of Stomach, Kj: Knee Joint, Ld: Lumen of Duodenum, LlL: Left Lobe of Liver, RT: Right Tibia, MT: Meta Tarsus, P: Pancreas, Ph: Phalanges, RF: Right Fibula, RlL: Right Lobe of Liver, Si: Small Intestine, T:Tarsus.
Labels: Cp: Cartilage Primordium of Basisphenoid, Ll: Left Lung, Sc: Spinal Cord, Cp Cv: Cartilage Primordium of Centrum of Eighth Cervical Vertibra, LC: Left Clavicle, LSm: Left Submandibular gland, LT: Lumen of Trachea, Op: Oesiphagus, Ps: Pectoralis Super Ficialis Muscle, RSm: Right Submandibular gland, RsV: Right Sub Clavian Vein, Sc: Spinal Cord, Cp Tv: Catilage Primordium of Upper Thoracic Vertebral Body, HPv: Hepatic Portal Vein, LlL: Left Lobe of Liver, Pc: Pleural Cavity, Ri: Ribs, RlL: Right Lobe of Liver, Ta: Trachea.
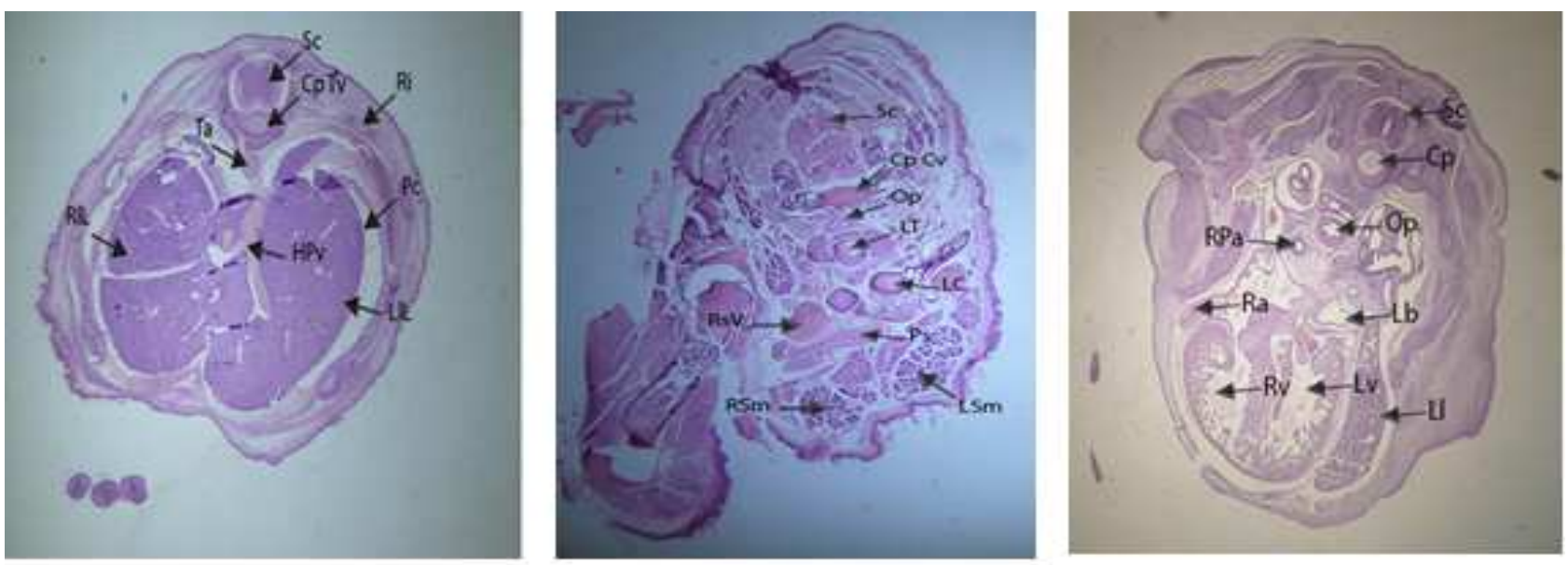

Figure 10: : Photomicrographs of fetuses from medium dose + antidote group through hepatic and cardiac regions
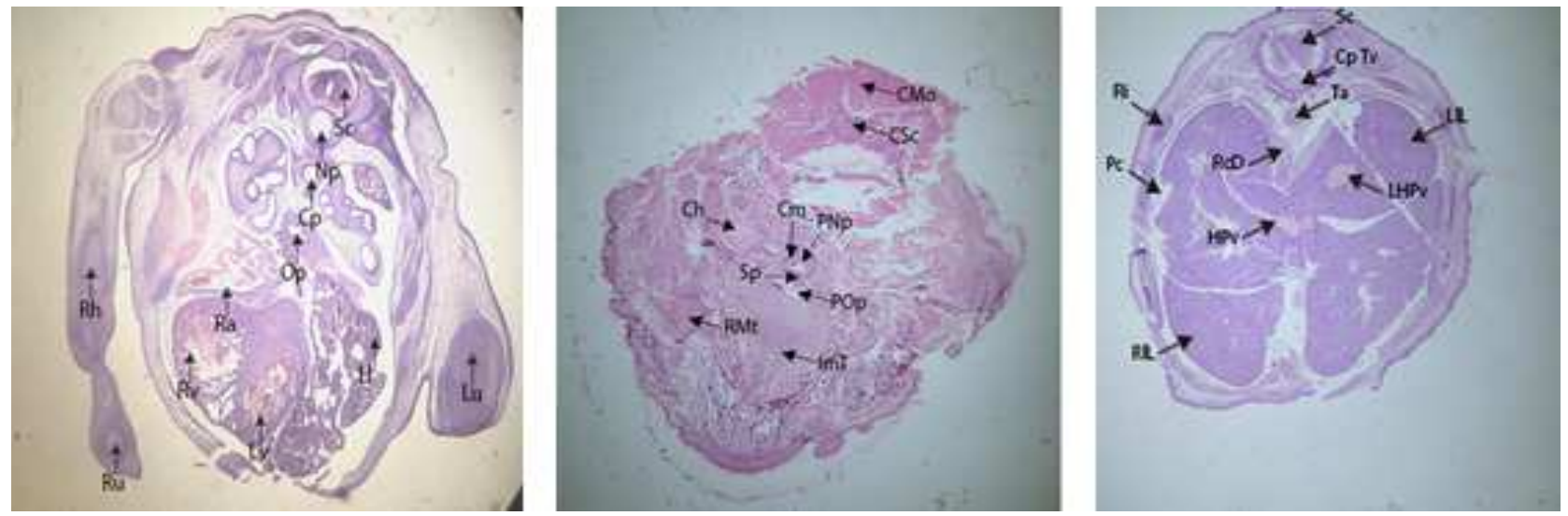

Figure 11: Cross sections of fetuses obtained from mothers from high dose + antidote group abdominal region.

Labels: Cp: Cartilage Primordium of Basisphenoid, Ll: Left Lung, Lu: Left Ulna, Lv: Left Ventricle, Np: Nucleus Pulposus, Op: Oesiphagus, Ra: Right Atrium, Rh: Right Humerus, Ru: Right Ulna, Rv: Right Ventricle, Sc: Spinal Cord, Ch: Cochlea, Cm: Cephalic Mesenchyme, CMo: Caudal part of Medulla Oblongata, CSc: Clivus, ImT: Intrinsic Muscle of Tongue, PNp: Posterior Nasopharynx, POp: Posterior Part of Pharynx, RMt: Right Molar Tooth, Sp: Soft
Palate, Cp Tv: Catilage Primordium of Upper Thoracic Vertebral Body, HPv: Hepatic Portal Vein, LlL: Left Lobe of Liver, LHPv: Left Hepatic Portal Vein, Pc: Pleural Cavity, RcD: Right Crus of Diaphragm, Ri: Ribs, RIL: Right Lobe of Liver, Ta: Trachea

High Dose (3.30 $\mu$ g/ g B.wt.) + Antidote Group: This exposure group show many abnormalities like thickened 
myocardium and degenerated lung tissue indicating congenital emphysema.

In all the exposure as well as antidote groups, liver seem to be normal with respect to histology and anatomy. The lungs in control, vehicle control and low dose $(1.65 \mu \mathrm{g} / \mathrm{g}$ B.wt.) groups were normal in their gross structures, while small lesions were observed in medium and high dose $(3.30 \mu \mathrm{g} / \mathrm{g}$ B.wt.) groups (fig. 11). Atenolol like that of other $\beta$-blockers can cross the Placental barrier and its level in maternal and fetal blood is more or less equal (Rasanen \& Jouppila, 1995). Cardiac function and umbilico-placental circulation of fetus are directly affected by atenolol. B-adrenergic receptors are present in placenta and umbilical vessels and atenolol causes reduced umbilical blood flow in humans. Thus placental weight is significantly reduced after maternal atenolol treatment during pregnancy (Montan et al., 2009). This decrease is correlated with intrauterine growth retardation (IUGR), anemia, oxidative stress and lower birth weight in human and rodent species independent of gestational age (Lip et al., 1997).

Conclusion: Atenolol can be used in pregnancy however there is insufficient data to prove its safety during first trimester. The present study revealed that the administration of atenolol during organo-genetic period can cause skeletal and histopathological deformities in developing mice embryos, which can be reduced by using Pomegranate juice.

If drug therapy is necessary, then to minimize risks and maintain maternal-fetal health fresh punica granatum juice should be used.

Acknowledgments: Authors are thankful to Department of Zoology, University of the Punjab, Lahore for providing research facilities.

Conflict of interest Statement: Authors declares no any kind of financial and authorship conflict of interest.

\section{REFERENCES}

Alexander, J. M. and K. L. Wilson (2013). Hypertensive emergencies of pregnancy. Obstet. and Gynecol. 40(1): 89-101. https://doi.org/10.1016/j.ogc.2012.11.008

Andrade, S. E., J. H. Gurwitz, R. L. Davis, K. A. Chan, J. A. Finkelstein, K. Fortman, ... R. Platt (2004). Prescription Drug Use in Pregnancy. Am. J. Obstet. Gynecol. 191(2): 398-407. https://doi.org/10.1016/j.ajog.2004.04.025

Aviram, M. (2004). Pomegranate juice consumption for 3 years by patients with carotid artery stenosis reduces common carotid intima-media thickness, blood pressure and LDL oxidation. Clin. Nutr. 23(3):423-33.

Aviram, M. and M. Rosenblat (2012). Pomegranate Protection against Cardiovascular Diseases. In
Evidence-based complementary and alternative medicine: eCAM (2012).

Awari, D. M., V. M. Mute, and B. B. Thube (2009). Cardiotonic Activity from the Fruit Juice of Punica Granatum. J. Pharm. Res. 2(2), 182-184.

Baker J. R. J. (1958). Histochem. Cytochem.: Official. J. Histochem. Soc. 6:303-308.

Bayliss, H., D. Churchill, M. Beevers, and D. G. Beevers (2002). Anti-Hypertensive Drugs in Pregnancy and Fetal Growth: Evidence for "Pharmacological Programming" in the First Trimester. Hypertension in Pregnancy. 21(2): 161-174. https://doi.org/10.1081/PRG 120013785

Butters, L., S. Kennedy, and P. C. Rubin (1990). Atenolol in Essential Hypertension During Pregnancy. British. Med. J. 301(6752): 587-589. https://doi.org/10.1136/bmj.301.6752.587.

Chobanian, A. V., G. L. Bakris, H. R. Black, W. C. Cushman, L. A. Green, J. L. Izzo, .... E. J. Roccella (2003) Seventh Report of the Joint National Committee on Prevention, Detection, Evaluation, and Treatment of High Blood Pressure. Hypertension. 42(6):1206-1252. doi: 10.1161/01.HYP.0000107251.49515.c2

Fitzgerald, J. D., R. Ruffin, K. G. Smedstad, R. Roberts, and J. McAinsh (1978). Studies on the Pharmacokinetics and Pharmacodynamics of Atenolol in Man. Eur. J. Clin. Pharmacol. 13(2):81-89.

Freyer, A. M. (2009). Drugs in Pregnancy and Lactation: A Reference Guide to Fetal and Neonatal Risk. Obstet. Med. 2(2): 89-89.

Hind, N. M. and E. A. Sara (2014). "Management of Hypertensive Disorders in Pregnancy." Women's Health. 10(4): 385-404.

Isla S. M., B. W. Ian and R. C. John (2005). Churchill's Pocketbook of Hypertension. Ist Ed. Churchill Livingstone London, 140p

James, P. A., S. Oparil, B. L. Carter, W. C. Cushman, C. Dennison-Himmelfarb, J. Handler, ... E. Ortiz (2014). 2014 Evidence-Based Guideline for the Management of High Blood Pressure in Adults: Report from the Panel Members Appointed to the Eighth Joint National Committee (JNC 8)2014 Guideline for Management of High Blood Pressure.JAMA.311(5):507-520. https://doi.org/10.1001/jama.2013.284427

Kawamura, S., A. Hirohashi, T. Kato, and M. Yasuda (1990). Bone-Staining Technique for Fetal Rat Specimens without Skinning and Removing Adipose Tissue. Congenital. Anomalies. 30: 9395.

https://doi.org/10.1111/j.17414520.1990.tb0049 8.x 
Lip, G. Y. H., M. Beevers, D. Churchill, L. M. Shaffer, and D. G. Beevers (1997). Effect of Atenolol on Birth Weight. Am. J. Cardio. 79(10): 14361438. https://doi.org/10.1016/S00029149(97)00163-XM.

Lydakis, C., G. Y. H. Lip, M. Beevers, and D. G. Beevers (1999). Atenolol and Fetal Growth in Pregnancies Complicated by Hypertension. Am. J. Hypertension. 12(6): 541-547. https://doi.org/10.1016/s0895-7061(99)00031-x

Mancia, G., S. Laurent, E. Agabiti-Rosei, M. Burnier, M. J. Caulfield, et al., 2009. Reappraisal of European guidelines on hypertension management: a European Society of Hypertension Task Force document. J. Hypertens. 27(11):2121-2158.

McCormack, T., T. Krause, and N. O'Flynn, (2012). Management of hypertension in adults in primary care: NICE guideline. British. J. Gen. Practice. 62(596): 163-164.

Montan, S., I. Ingemarsson, K. Marsal, and N. O. Sjoberg (1992). "Randomised controlled trial of atenolol and pindolol in human pregnancy: effects on fetal haemodynamics." BMJ. 304(6832): 946949.

Moussa, H. N., S. E. Arian, and B. M. Sibai (2014). Management of Hypertensive Disorders in Pregnancy. Women's Health, 10(4): 385-404.
https://doi.org/10.2217/WHE.14.32

Räsänen, J. and P. Jouppila (1995). "Uterine and fetal hemodynamics and fetal cardiac function after atenolol and pindolol infusion. A randomized study." Euro. J. Obstet. Gyn. Reprod. Biol. 62(2): 195-201.

Reynolds, B., L. Butters, J. Evans, T. Adams, and P. C. Rubin (1984). First year of life after the use of atenolol in pregnancy associated hypertension. Arch. Dis. Child. 59(11): 1061-1063.

Ravindra Babu, P., H. P. L. Peter, M. Ankaiah, P. Hemanth Sairam, and M. Ramesh (2012). Positive Inotropic Actvity of Aqueous Extract of Pericarp of Punica Granatum on Isolated Frog's Heart. Int. J. Pharm. and Pharmaceutic. Sci. 4(SUPPL.3): 95-98.

Tabacova, S. C. A. Kimmel, K. Wall, and D. Hansen (2003). Atenolol Developmental Toxicity: Animal-to-Human Comparisons. Birth Defects Research Part A - Clin. Mol. Teratol. 67(3): 181-192. https://doi.org/10.1002/bdra.10011

The atlas of mouse development, by M.H. Kaufman, Academic Press, San Diego, CA, 1992, 512 pp, https://doi.org/10.1002/mrd.1080370119

The Seventh Report of the Joint National Committee on Prevention, Detection, Evaluation, and Treatment of High Blood Pressure: the JNC 7 report. (2003). JAMA. 289(19):2560-72. 